\title{
The neutrophil-to-lymphocyte ratio prechemo- therapy and postchemotherapy as a prognostic marker in metastatic gastric cancer
}

\author{
Hyunho Kim ${ }^{1}$, Sang Mi Ro ${ }^{2}$, Ji Hyun Yang ${ }^{2}$, Joon Won Jeong ${ }^{2}$, Ji Eun Lee ${ }^{2}$, Sang Young Roh ${ }^{2}$, and
} In-Ho Kim ${ }^{2}$

\begin{abstract}
${ }^{1}$ Department of Internal Medicine, Korea Military Academy Hospital, Seoul; ${ }^{2}$ Division of Medical Oncology, Department of Internal Medicine, College of Medicine, Seoul St. Mary's Hospital, The Catholic University of Korea, Seoul, Korea
\end{abstract}

Received: September 11, 2016 Revised : December 20, 2016 Accepted: January 18, 2017

\author{
Correspondence to \\ In-Ho Kim, M.D. \\ Department of Internal \\ Medicine, College of Medicine, \\ Seoul St. Mary's Hospital, \\ The Catholic University of Korea, \\ 222 Banpo-daero, Seocho-gu, \\ Seoul 06591, Korea \\ Tel: $+82-2-2258-6046$ \\ Fax: $+82-2-599-3589$ \\ E-mail:ihkmd@catholic.ac.kr
}

Background/Aims: Markers of inflammation have been associated with outcomes in various cancers. The purpose of this study was to evaluate whether systemic inflammatory markers and their fluctuations can predict survival and chemotherapy response in patients with metastatic gastric cancer (mGC).

Methods: We retrospectively reviewed the records of 502 patients who received first-line palliative chemotherapy for mGC between 2007 and 2013. The neutrophil-to-lymphocyte ratio (NLR) and modified Glasgow prognostic score (mGPS) were assessed before and after chemotherapy to evaluate their association with survival. The NLR values were categorized into two groups based on a cut-off value of 3 ; mGPS values were classified as high versus low.

Results: High prechemotherapy NLR was significantly associated with poor overall survival on univariate analysis $(p=0.002)$. On multivariate analysis, high prechemotherapy NLR (hazard ratio, 1.43; $p<0.001$ ) was an independent prognostic factor for poor overall survival. However, the prechemotherapy mGPS was not significantly associated with survival. Continuously high NLR or a shift to high NLR postchemotherapy was associated with poor chemotherapy response as well as survival, while NLR reduction was associated with a good response (linear by linear association, $p<0.001$ ) and a favorable prognosis.

Conclusions: Prechemotherapy NLR can be used as a prognostic factor in mGC, while the postchemotherapy NLR value may predict the chemotherapeutic response and prognosis. In contrast, mGPS has limited prognostic utility in mGC.

Keywords: Biomarkers; Neutrophils; Stomach neoplasms; Drug therapy; Survival

\section{INTRODUCTION}

Gastric cancer is the fifth most common cancer and the third most common cause of cancer-related deaths worldwide [1]. Gastric cancer is often diagnosed at an advanced stage $[2,3]$ and has a poor prognosis, especially in patients with metastatic gastric cancer (mGC) $[1,4]$. The Trastuzumab for Gastric Cancer (ToGA) trial showed that chemotherapy combined with trastuzum$\mathrm{ab}$, a monoclonal antibody against human epidermal growth factor receptor 2 (HER2), slightly but significantly improved survival in HER2-positive mGC compared to chemotherapy alone [5]. However, chemotherapy remains the mainstream first-line treatment for mGC, and the overall survival (OS) of mGC averages less than 1 year $[2,4,6-9]$. Therefore, investigators have continued 
to search for prognostic markers that can also predict responses to chemotherapy.

Several recent studies showed that inflammatory markers can be used to predict prognosis in patients with gastric cancer and in chronic obstructive pulmonary disease [10-17], as inflammation has been shown to play a role in tumor progression [18-20]. Tumor cells produce inflammatory cytokines that attract various leukocytes, such as neutrophils and lymphocytes [19]; these inflammatory cytokines can enhance tumor proliferation, angiogenesis, invasion, and metastasis $[19,20]$. Furthermore, the neutrophil-to-lymphocyte ratio (NLR) can indicate the presence of neutrophilia as well as lymphopenia related to the suppression of cell-mediated immunity in patients with cancer $[18,19]$. Mohri et al. [10] reported that preoperative NLR could be a postoperative prognostic marker in patients with curable gastric cancer; pretreatment NLR was also associated with survival in mGC $[12,14]$. Aside from NLR, the modified Glasgow prognostic score (mGPS) has been studied as another inflammation-based marker to predict prognosis. The mGPS is calculated using C-reactive protein (CRP) and serum albumin levels, and can reflect both elevated CRP and hypoalbuminemia [15]; of note, some studies reported that mGPS was significantly associated with survival in gastric cancer $[11,13,14]$.

Both NLR and mGPS have mainly been studied at the time of pretreatment or in early stage gastric cancer $[10,12,13,15,21]$. This study first aimed to determine if pretreatment NLR and mGPS values are predictive of survival and chemotherapy response in patients with mGC. Additionally, we assessed whether postchemotherapy NLR and mGPS values are useful prognostic factors for survival and chemotherapy response in these patients.

\section{METHODS}

\section{Study population}

This was a retrospective study that was approved by the Institutional Review Board of the Seoul St. Mary's Hospital of the Catholic University of Korea (KC16RISIo113). In carrying out the study, informed consents were not required because of simple medical record reviews. The medical records of 502 patients who received first-line palliative chemotherapy for $\mathrm{mGC}$ at the hospital between 2007 and 2013 were reviewed. All patients were diagnosed by pathologic confirmation from a primary gastric cancer biopsy sample or from surgical specimens. Histological types were classified into differentiated (well or moderate differentiation) or undifferentiated (poor differentiation, mucinous adenocarcinoma, signet ring cell adenocarcinoma, or poorly cohesive carcinoma) types. Metastases were classified as recurrent versus initially diagnosis. Patients who had a concomitant infection, steroid treatment, or other conditions that affect their systemic inflammation marker levels, such as autoimmune disease, were excluded from this study.

\section{Treatment and evaluation of the tumor response}

Palliative chemotherapy was administered to all patients after the diagnosis of mGC. Platinum-based chemotherapy $(\mathrm{n}=398,79.3 \%)$ was used most frequently, and FOLFOX (oxaliplatin, 5-fluorouracil, and leucovorin) was the most common regimen. The chemotherapy regimen was selected at the discretion of the treating clinician. Prechemotherapy blood tests, including complete blood count (CBC) and blood chemistry (BC) testing for evaluating albumin and CRP, were conducted 4 to 24 hours before commencing chemotherapy. CBCs and BC tests were also performed every $8 \pm 2$ weeks after commencing chemotherapy. All patients underwent chest and abdomen computed tomography. Baseline imaging was performed 1 week before initiation of chemotherapy, and follow-up images were acquired every $8 \pm 2$ weeks after commencing chemotherapy. Radiological changes were evaluated using the Response Evaluation Criteria for Solid Tumors (RECIST) version 1.1 [22]. Objective response was defined as complete response (CR) or partial response (PR), while disease control was defined as CR, $\mathrm{PR}$, or stable disease. Postchemotherapy blood testing, including $\mathrm{CBC}$ and $\mathrm{BC}$, was conducted at the same time as follow-up images were acquired for chemotherapy response evaluation.

\section{Measuring and defining levels of NLR and mGPS}

In this study, we assessed NLR and mGPS as systemic inflammation markers. NLR is the neutrophil count divided by the lymphocyte count, and was classified as high (NLR $\geq 3.0)$ and low (NLR < 3). mGPS was calculated using CRP and serum albumin levels: an elevated CRP 
levels (> $1 \mathrm{mg} / \mathrm{dL}$ ) and hypoalbuminemia $(<3.5 \mathrm{~g} / \mathrm{dL})$ was designated as 2 points, elevated CRP and normal albumin levels denoted 1 point, and CRP levels $\leq 1 \mathrm{mg} / \mathrm{dL}$ with normal albumin levels indicated o points. mGPS was classified into two groups: high (a score of 2 ) and low (a score of o or 1). There were four categories of change in NLR and $m$ GPS values between the prechemotherapy and postchemotherapy periods: low to low, high to low, low to high, and high to high; these were designated as A, B, C, and D, respectively.

\section{Statistical analysis}

OS and progression-free survival (PFS) were defined as the date from starting first-line palliative chemotherapy to death and disease progression, respectively. Survival curves were calculated using the Kaplan-Meier method, and the log-rank test was used to compare each survival
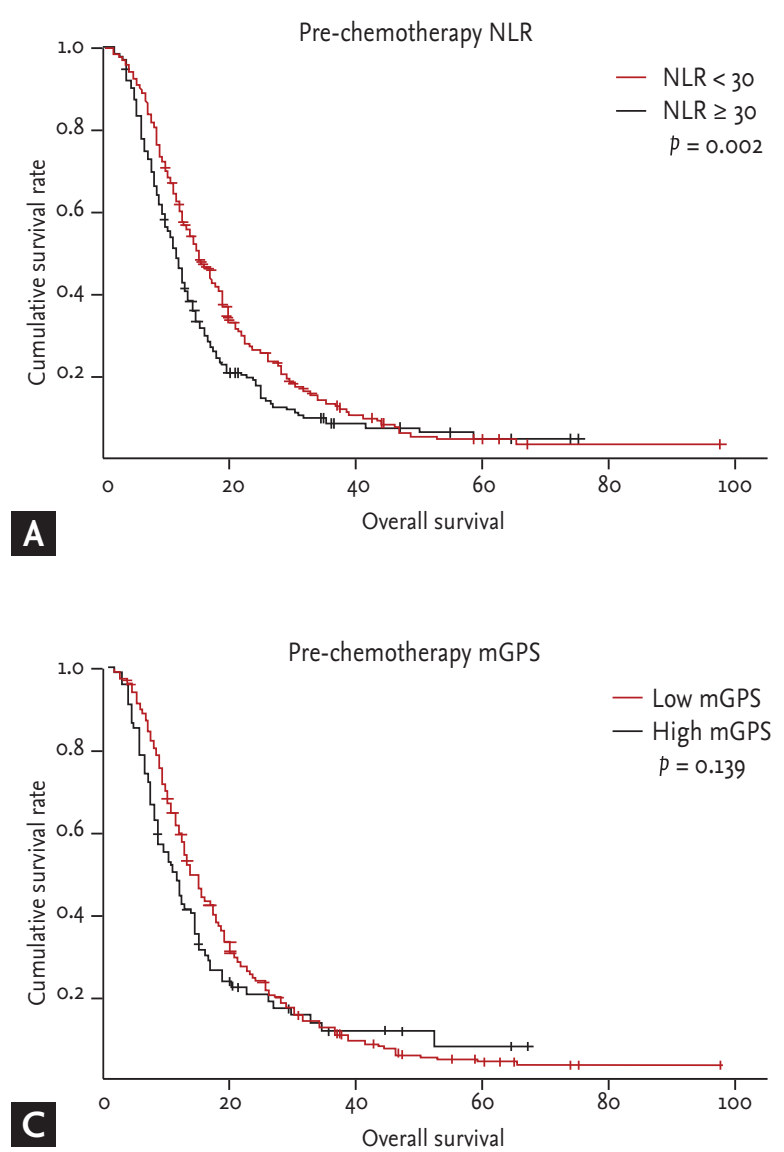

curve. The Cox proportional hazards method was used to perform multivariate analysis to evaluate the significance of the prognostic variables. The chi-square test was used to determine the significance of the correlations between NLR and chemotherapy response, and linear by linear association was used to assess the trend between change patterns in NLR and chemotherapy response. All statistical analyses were performed using the SPSS software version 22.o (IBM Co., Armonk, NY, USA), and a two-sided $p<0.05$ was considered statistically significant.

\section{RESULTS}

\section{Patient characteristics}

This study included a total of 502 patients; their char-
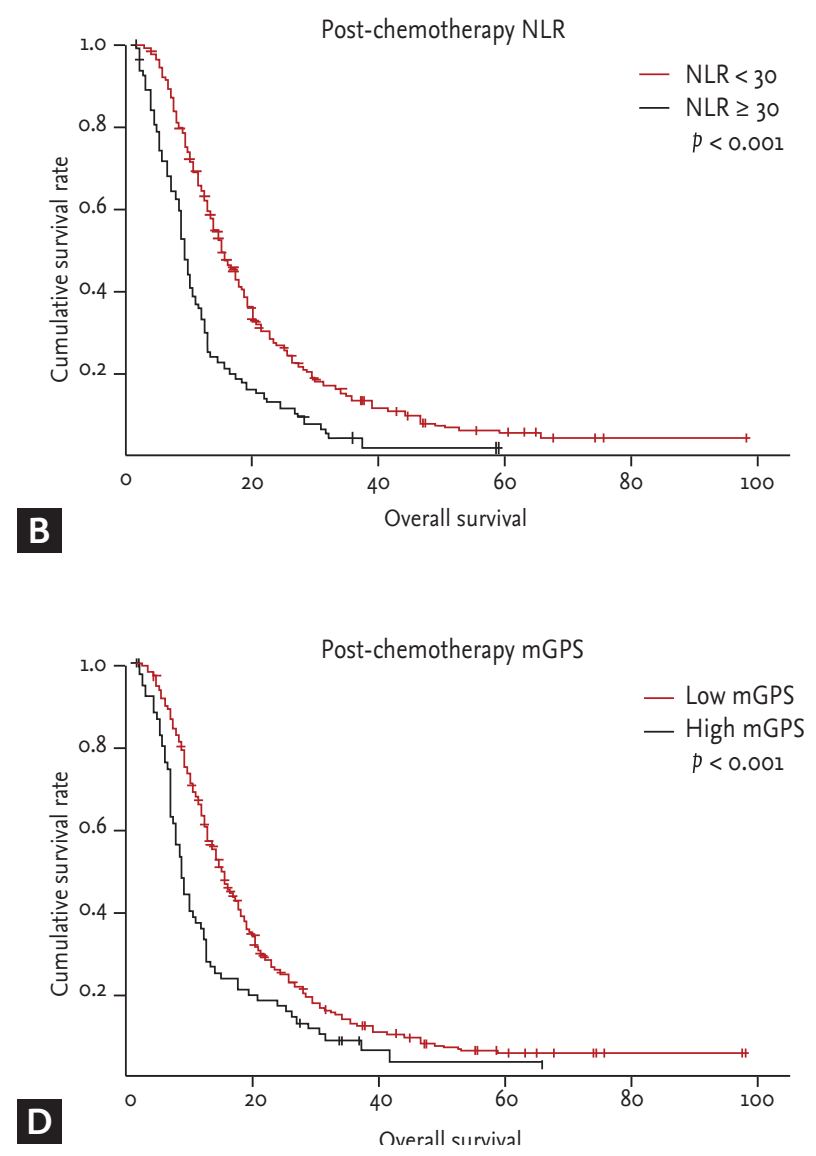

Figure 1. Survival curves depending on the inflammatory markers, obtained by using the Kaplan-Meier method. (A, B) The survival curves of high prechemotherapy/postchemotherapy neutrophil-to-lymphocyte ratio (NLR) were significantly different from the low NLR ( $p=0.002$ and $p=0.001$, respectively) (C) The survival curves of high prechemotherapy modified Glasgow prognostic score (mGPS) was not significantly different from the low mGPS $(p=0.139)$. (D) The survival curve of high postchemotherapy mGPS was significantly different from the low $\operatorname{mGPS}(p=0.001)$. 
Table 1. Patients baseline

\begin{tabular}{|c|c|}
\hline Characteristic & Value \\
\hline All patients & 502 \\
\hline Age, yr & $58(20-81)$ \\
\hline$<65$ & $333(66.3)$ \\
\hline$\geq 65$ & $169(33.7)$ \\
\hline \multicolumn{2}{|l|}{ Sex } \\
\hline Female & $202(40.2)$ \\
\hline Male & $300(59.8)$ \\
\hline \multicolumn{2}{|l|}{ Histological type } \\
\hline Differentiated ${ }^{\mathrm{a}}$ & $177(35.3)$ \\
\hline Undifferentiated $^{\mathrm{b}}$ & $325(64.7)$ \\
\hline \multicolumn{2}{|l|}{ Metastatic presentation } \\
\hline Recurrence & $146(29.1)$ \\
\hline Initial stage & $356(70.9)$ \\
\hline \multicolumn{2}{|l|}{ No. of metastatic organs } \\
\hline 1 & $202(40.2)$ \\
\hline$\geq 2$ & $300(59.8)$ \\
\hline \multicolumn{2}{|l|}{ Peritoneal carcinomatosis } \\
\hline No & $261(52.0)$ \\
\hline Yes & $241(48.0)$ \\
\hline \multicolumn{2}{|l|}{ First line chemotherapy } \\
\hline Platinum based regimen & $398(79 \cdot 3)$ \\
\hline Non-platinum based regimen & $104(20.7)$ \\
\hline \multicolumn{2}{|l|}{$\begin{array}{l}\text { Prechemotherapy } \\
\text { laboratory parameter }\end{array}$} \\
\hline WBC, $1,000 / \mathrm{mm}^{3}$ & $6,680(1,580-31,820)$ \\
\hline ANC, $1,000 / \mathrm{mm}^{3}$ & $4,048(480-27,057)$ \\
\hline Lymphocyte, $1,000 / \mathrm{mm}^{3}$ & $1,588(231-3,796)$ \\
\hline Neutrophil-to-lymphocyte ratio & $2.51(0.41-45.81)$ \\
\hline$<3.00$ (Low) & $317(63.1)$ \\
\hline$\geq 3.00$ (High) & $185(36.9)$ \\
\hline Albumin, g/dL & $3.80(2.10-5.10)$ \\
\hline $\mathrm{CRP}, \mathrm{mg} / \mathrm{dL}$ & $0.80(0.01-35.00)$ \\
\hline \multicolumn{2}{|l|}{ Modified Glasgow prognostic score } \\
\hline o or 1 score (low) & $412(82.1)$ \\
\hline 2 score (high) & $90(17.9)$ \\
\hline
\end{tabular}

Values are presented as median (range) or number (\%). WBC, white blood cell; ANC, absolute neutrophil count; CRP, C-reactive protein.

${ }^{a}$ Differentiated histological type included well differentiation and moderate differentiation.

${ }^{\mathrm{b}}$ Undifferentiated histological type included poor differentiation, signet ring cell adenocarcinoma and mucinous adenocarcinoma. acteristics are listed in Table 1. The median patient age was 58 years with a range of 20 to 81 years, and included 300 men and 202 women. Of these patients, 441 died; the median survival time was 12.85 months. The median NLR was 2.51 (range, 0.41 to 45.81 ), with 185 and 317 patients classified into the high NLR ( $\geq 3)$ and low NLR (< 3) groups, respectively. A high mGPS (score, 2) was found in 90 patients.

\section{Prognostic value of prechemotherapy/postchemo- therapy NLR and mGPS on survival}

The impact of clinicopathological factors and systemic inflammation markers on OS and PFS were analyzed; the results are listed in Table 2. Univariate analyses of OS and PFS showed that detecting metastasis at initial diagnosis (OS: hazard ratio [HR], 1.55, $p<0.001$; PFS: HR, $1.49, p<0.001)$, an undifferentiated histological type (OS: HR, 1.43, $p<0.001$; PFS: HR, 1.28, $p=0.005$ ), peritoneal carcinomatosis (OS: HR, 1.42, $p<0.001$ ), and metastasis to two or more organs (OS: HR, 1.65, $p<0.001$; PFS: HR, $1.58, p<0.001)$ were significantly associated with poor survival. On multivariate analysis, an age of 65 years or older (OS: HR, 1.23, $p=0.044$ ), detecting metastasis at diagnosis (OS: HR, 1.44, $p=0.001$; PFS: HR, 1.36, $p=$ 0.005), undifferentiated histological type (OS: HR, 1.55, $p<0.001$; PFS: HR, 1.33, $p=0.005)$, and metastasis to two or more organs (OS: HR, 1.56, $p<0.001$; PFS: HR, 1.48, $p<0.001)$ were significant independent prognostic factors. (Supplementary Table 1). The survival curve of high prechemotherapy NLR was significantly different from that of the low NLR group on univariate analysis (median OS: 14.90 months vs. 11.47 months, $p=0.002$; median PFS: 7.50 months vs. 5.07 months, $p=0.002$, respectively) (Fig. 1, Supplementary Table 2, and Supplementary Fig. 1). On multivariate analysis, high prechemotherapy NLR (OS: HR, 1.43, p< 0.001; PFS: HR, 1.42, p< 0.001) was also a significant independent prognostic factor (Supplementary Table 1). On the other hand, the survival curve of high prechemotherapy mGPS was not different from that of the low mGPS on univariate analysis (OS: $p=$ 0.139; PFS: $p=0.129$ ) (Fig. 1, Supplementary Table 2, and Supplementary Fig. 1), and mGPS was not an independent prognostic factor on multivariate analyses (Table 2). Following chemotherapy, both NLR and mGPS had significantly different survival curves when comparing the high and low groups on univariate analysis $(p<0.001$ 
Table 2. Univariate and multivariate analyses for prechemotherapy overall survival

\begin{tabular}{|c|c|c|c|c|c|c|}
\hline \multirow{2}{*}{ Variable } & \multicolumn{3}{|c|}{ Univariate } & \multicolumn{3}{|c|}{ Multivariate } \\
\hline & HR & $95 \% \mathrm{CI}$ & $p$ value & HR & $95 \% \mathrm{CI}$ & $p$ value \\
\hline \multicolumn{7}{|l|}{ Age, yr } \\
\hline$<65$ & 1.00 & & & 1.00 & & \\
\hline$\geq 65$ & 1.08 & $0.89-1.32$ & 0.434 & 1.23 & $1.01-1.51$ & 0.044 \\
\hline \multicolumn{7}{|l|}{ Sex } \\
\hline Female & 1.00 & & & & & \\
\hline Male & 1.00 & $0.83-1.22$ & 0.967 & & NS & \\
\hline \multicolumn{7}{|l|}{ Detecting metastasis } \\
\hline Recurrence & 1.00 & & & 1.00 & & \\
\hline Initial diagnosis & 1.55 & $1.26-1.91$ & $<0.001$ & 1.44 & $1.17-1.79$ & 0.001 \\
\hline \multicolumn{7}{|l|}{ Histological type } \\
\hline Differentiated & 1.00 & & & 1.00 & & \\
\hline Undifferentiated & 1.43 & $1.17-1.74$ & $<0.001$ & 1.55 & $1.26-1.91$ & $<0.001$ \\
\hline \multicolumn{7}{|c|}{ Peritoneal carcinomatosis } \\
\hline No & 1.00 & & & & & \\
\hline Yes & 1.42 & $1.18-1.72$ & $<0.001$ & & NS & \\
\hline \multicolumn{7}{|l|}{ No. of metastatic organs } \\
\hline 1 & 1.00 & & & 1.00 & & \\
\hline$\geq 2$ & 1.65 & $1.36-2.00$ & $<0.001$ & 1.56 & $1.28-1.91$ & $<0.001$ \\
\hline \multicolumn{7}{|l|}{ First line chemotherapy } \\
\hline Cisplatin based & 1.00 & & & & & \\
\hline Non-cisplatin based & 0.99 & $0.79-1.24$ & 0.921 & & NS & \\
\hline \multicolumn{7}{|l|}{ Prechemotherapy NLR } \\
\hline$<3.0$ & 1.00 & & & 1.00 & & \\
\hline$\geq 3.0$ & 1.35 & $1.11-1.64$ & 0.003 & 1.43 & $1.17-1.73$ & $<0.001$ \\
\hline \multicolumn{7}{|l|}{ Prechemotherapy mGPS } \\
\hline Low & 1.00 & & & & & \\
\hline High & 1.21 & $0.94-1.55$ & 0.140 & & NS & \\
\hline
\end{tabular}

HR, hazard ratio; CI, confidence interval; NS, not significant; NLR, neutrophil-to-lymphocyte ratio; mGPS, modified Glasgow prognostic score.

for all comparisons) (Fig. 1 and Supplementary Table 3).

\section{Correlation of change patterns in NLR with chemo- therapy response and survival}

This study assessed the proportion of chemotherapy response depending on change patterns in NLR before and after chemotherapy; these changes are shown in Table 3. The total objective response rate (ORR) was 51.2\%, and the total disease control rate (DCR) was $86.9 \%$. The proportions of ORR were significantly different depending on the NLR change pattern, classified into groups A to $\mathrm{D}$ as described in 'Methods' $\left(\chi^{2}=24.791, p<0.001\right)$.
The proportions of DCR were also significantly different depending on the NLR change pattern $\left(\chi^{2}=58.569\right.$, $p$ $<0.001)$. Decreasing trends for both ORR and DCR were observed when the NLR change pattern shifted toward group D (linear by linear association value, 20.804 and 45.748, respectively; both $p<0.001$ ) (Table 3).

The survival curves of group A (median OS, 16.93 months), group B (median OS, 16.93 months), group C (median OS, 13.13 months), and group D (median OS, 8.73 months) were significantly different from each other $(p<0.001)$ on univariate analysis (Fig. 2 and Supplementary Table 4). On multivariate analysis, groups B, C, 
and D were significantly associated with poorer survival compared to group A (Table 4).

\section{Prognostic value of prechemotherapy NLR accord- ing to HER2 expression}

The association between prechemotherapy NLR and survival was assessed according to HER2 expression status in 193 patients with available HER2 expression data (the remaining patients had been registered before 2010, and their HER2 data were unavailable). There were 161 HER2-negative patients for whom univariate analyses of OS and PFS revealed that prechemotherapy NLR was significantly associated with poor survival (OS: $p=0.002$; PFS: $p=0.006$ ) (Supplementary Table 5). In 32 patients who were HER2-positive, the prechemotherapy NLR was not associated with survival on univariate analyses of OS and PFS (OS: $p=0.105$; PFS: $p=0.450$ ). However, multivariate analyses showed that prechemotherapy NLR was significantly associated with poor survival (OS: $p=0.001 ;$ PFS: $p=0.007$ ) (Supplementary Table 6). There was no significant correlation between prechemotherapy NLR and HER2 expression (Pearson correlation coefficient, $0.06 ; p=0.410$ ).

\section{DISCUSSION}

Our study showed that NLR was a useful prognosticator in mGC, while mGPS was not associated with survival. The reason for the correlation of NLR with prognosis

Table 3. Correlation of change patterns in NLR with chemotherapy response

\begin{tabular}{|c|c|c|c|c|c|c|c|}
\hline \multirow[b]{2}{*}{ Variable } & \multirow[b]{2}{*}{ No. (\%) } & \multicolumn{4}{|c|}{ NLR change pattern } & \multirow{2}{*}{$\begin{array}{c}\text { Chi-square, } \\
\text { p value }\end{array}$} & \multirow{2}{*}{$\begin{array}{c}\text { Linear by } \\
\text { linear }\end{array}$} \\
\hline & & $\begin{array}{l}\text { Low to low } \\
\qquad(\mathrm{n}=253)\end{array}$ & $\begin{array}{l}\text { High to low } \\
\qquad(\mathrm{n}=138)\end{array}$ & $\begin{array}{l}\text { Low to high } \\
\qquad(\mathrm{n}=64)\end{array}$ & $\begin{array}{l}\text { High to high } \\
\quad(\mathrm{n}=47)\end{array}$ & & \\
\hline CR & $82(16.3)$ & $48(19.0)$ & $21(15.2)$ & $7(10.9)$ & $6(12.8)$ & & \\
\hline PR & $175(34.9)$ & $100(39.5)$ & $54(39.1)$ & $12(18.8)$ & $9(19.1)$ & & \\
\hline $\mathrm{SD}$ & $179(35 \cdot 7)$ & $91(36.0)$ & $49(35 \cdot 5)$ & $21(32.8)$ & $18(38.3)$ & & \\
\hline $\mathrm{PD}$ & $66(13.1)$ & $14(5 \cdot 5)$ & $14(10.1)$ & $24(37.5)$ & $14(29.8)$ & & \\
\hline ORR & $257(51.2)$ & $148(58.5)$ & $75(54 \cdot 3)$ & $19(29.7)$ & $15(31.9)$ & $<0.001$ & $<0.001$ \\
\hline Non-ORR & $245(48.8)$ & $105(41.5)$ & $63(45 \cdot 7)$ & $45(70.3)$ & $32(68.1)$ & & \\
\hline DCR & $436(86.9)$ & $239(94.5)$ & $124(89.9)$ & $40(62.5)$ & $33(70.2)$ & $<0.001$ & $<0.001$ \\
\hline Non-DCR & $66(13.1)$ & $14(5 \cdot 5)$ & $14(10.1)$ & $24(37 \cdot 5)$ & $14(29.8)$ & & \\
\hline
\end{tabular}

Values are presented as number (\%).

NLR, neutrophil-to-lymphocyte ratio; CR, complete response; PR, partial response; $\mathrm{SD}$, stable disease; PD, progressive disease; ORR, objective response; DCR, disease control rate.
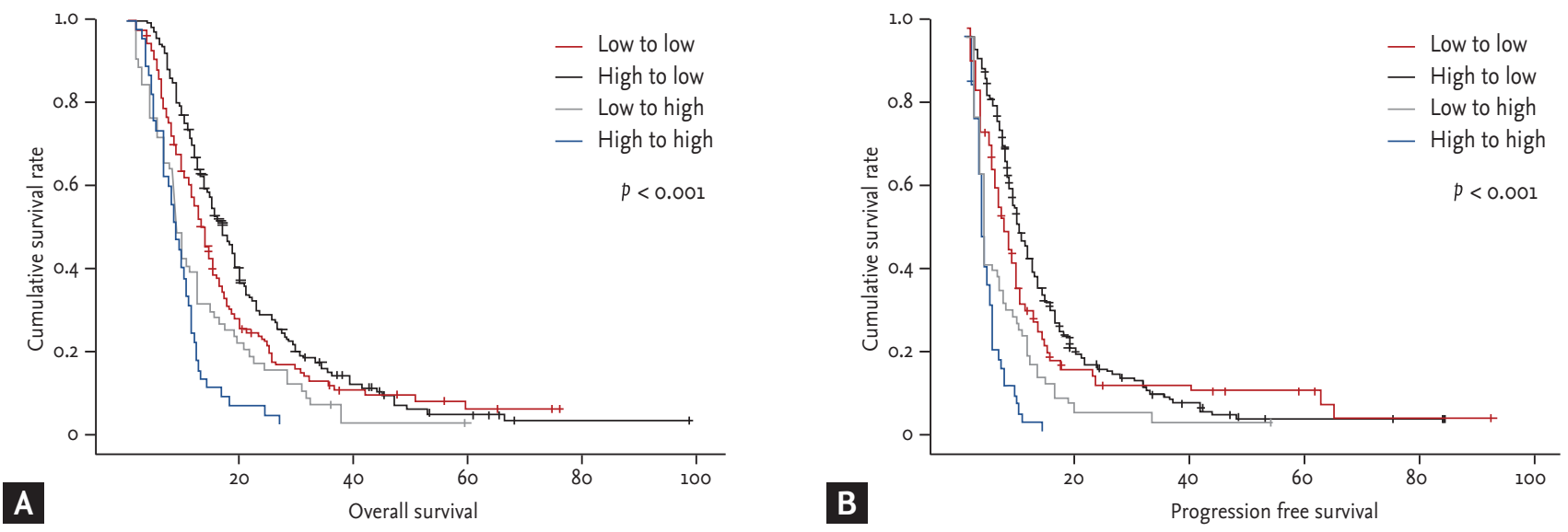

Figure 2. Survival curves depending on the change pattern of neutrophil-to-lymphocyte ratio (NLR). (A, B) According to the change pattern of NLR, the survival curves were significantly different from each other $(p<0.001$ for both). 
Table 4. Multivariate analysis for OS and PFS according to the change pattern of NLR

\begin{tabular}{|c|c|c|c|c|c|c|}
\hline \multirow{2}{*}{ Variable } & \multicolumn{3}{|c|}{ OS } & \multicolumn{3}{|c|}{ PFS } \\
\hline & HR & $95 \% \mathrm{CI}$ & $p$ value & HR & $95 \% \mathrm{CI}$ & $p$ value \\
\hline \multicolumn{7}{|l|}{ Age, yr } \\
\hline$<65$ & 1.00 & & & & & \\
\hline$\geq 65$ & 1.27 & $1.03-1.56$ & 0.022 & & NS & \\
\hline \multicolumn{7}{|l|}{ Histological type } \\
\hline Differentiated ${ }^{\mathrm{a}}$ & 1.00 & & & 1.00 & & \\
\hline Undifferentiated $^{\mathrm{b}}$ & 1.53 & $1.25-1.88$ & $<0.001$ & 1.33 & $1.09-1.62$ & 0.006 \\
\hline \multicolumn{7}{|c|}{ No. of metastatic organs } \\
\hline 1 & 1.00 & & & 1.00 & & \\
\hline$\geq 2$ & 1.51 & $1.23-1.84$ & $<0.001$ & 1.42 & $1.16-1.73$ & 0.001 \\
\hline \multicolumn{7}{|l|}{ Detecting metastasis } \\
\hline Recurrence & 1.00 & & & 1.00 & & \\
\hline Initial diagnosis & 1.41 & $1.13-1.75$ & 0.002 & 1.34 & $1.08-1.67$ & 0.007 \\
\hline NLR change pattern & & & $<0.001$ & & & $<0.001$ \\
\hline Low to low & 1.00 & & & 1.00 & & \\
\hline High to low & 1.35 & $1.08-1.70$ & 0.009 & 1.34 & $1.07-1.68$ & 0.012 \\
\hline Low to high & 1.83 & $1.38-2.44$ & $<0.001$ & 2.10 & $1.58-2.80$ & $<0.001$ \\
\hline High to high & 2.81 & $2.02-3.90$ & $<0.001$ & 3.71 & $2.65-5.20$ & $<0.001$ \\
\hline
\end{tabular}

OS, overall survival; PFS, progression-free survival; NLR, neutrophil-to-lymphocyte ratio; HR, hazard ratio; CI, confidence interval.

${ }^{a}$ Differentiated histological type included well differentiation and moderate differentiation.

${ }^{\mathrm{b}}$ Undifferentiated histological type included poor differentiation, signet ring cell adenocarcinoma, and mucinous adenocarcinoma.

in mGC has not yet been explained, although the relationship between inflammation and cancer is well documented [18-20,23]. Tumor cells closely interact with the extracellular matrix and with stromal cells around tumors [23,24]. This interaction contributes to the development of the tumor microenvironment, where stromal cells recruit various cytokine-producing inflammatory cells [19,24]. Fang and Declerck [24] described cancer cells and their microenvironment as the "malignant organ" that facilitates the tumorigenic process that includes proliferation, evasion of growth suppressors, resistance to apoptosis, development of replicative immortality, induction of angiogenesis, and activation of invasion and metastasis [23]. Among inflammatory cells, neutrophils particularly have a key role in facilitating cancer progression in the tumor microenvironment [25]. Neutrophils impact not only angiogenesis, migration, invasion, and metastasis, but also the suppression of immune responses against cancer cells [25]. They release various factors such as matrix metalloprotease 9 and vascular endothelial growth factor that recruit other inflammatory cells and promote cancer progression [19,25].

Lymphocytes are important extrinsic tumor suppressors [26]. They play a role in the detection of tumor cells, tumor infiltration, and direction of immune responses that eliminate these tumor cells $[18,26]$. In other words, a low lymphocyte count may reflect suppressed cell-mediated immunity against cancer [26]; conversely, a high T-cell count has been correlated with survival in various solid tumors [18]. Cho et al. [12] reported that a low absolute lymphocyte count was associated with longer survival in mGC $(p=0.050)$. Taken together, the NLR likely influences survival outcomes in mGC because of a combination of absolute neutrophil and lymphocyte counts. As expected, prechemotherapy NLR was definitely associated with OS and PFS in this study, as was postchemotherapy NLR.

The survival curves representing NLR change patterns before and after chemotherapy differed significantly 
from each other, as did the proportion of patients in whom response to chemotherapy was influenced by the NLR change pattern. A higher postchemotherapy NLR is associated with a worse OS, PFS, and chemotherapy response. When considering the tumor microenvironment, residual cancer burden after chemotherapy may be correlated with increased inflammatory cells, such as neutrophils. Thus, a shift toward a high NLR may predict poor response to chemotherapy in mGC. These data suggest that controlling inflammation in tumors may help improve the prognosis of $\mathrm{mGC}$, and measurement of NLR after chemotherapy could help to evaluate chemotherapy response when imaging studies prove inadequate. For example, if a patient without a measurable lesion exhibits reduced NLR, it could be regarded as a favorable response to chemotherapy.

HER2 overexpression is a poor prognostic factor $[27,28]$. Hence, we analyzed the correlation between prechemotherapy NLR and HER2 expression, but found no such association. The prechemotherapy NLR in HER2-positive patients was not significantly associated with survival, which was contrary to our expectation. However, the median survival of patients with high NLR (13.53 months) was shorter than that of patients with low NLR (15.73 months). There were 32 HER2-positive patients in our study who were divided into high NLR (19 patients) and low NLR (13 patients) groups; our results may have been different if more HER2-positive patients were included in the study.

In pursuing this investigation, it was difficult to determine an appropriate cut-off value for high versus low NLR; hence, the optimal cut-off value remains unclear. Most previous studies used an NLR of 3 or 3.5 as the cutoff in gastric cancer $[10,12,14,16]$. Cho et al. [12] and Hsieh et al. [14], who used an NLR cut-off of 3, showed that a high pretreatment NLR was significantly associated with poorer prognosis in $\mathrm{mGC}$; therefore, we similarly selected a cut-off value of 3 for dividing our population into high and low NLR groups. An NLR cut-off of 3.5 produced similar results insofar as prechemotherapy NLR being a significant prognostic factor $(p<0.001)$; however, an NLR cut-off of 3 was chosen instead of 3.5 to achieve a numerical balance between the high and low NLR groups.

Forrest et al. [29] suggested a new scoring system based on CRP and serum albumin levels, i.e., mGPS, to evaluate prognosis in non-small cell lung cancer. Since then, several studies have suggested that mGPS is a useful prognostic factor in various cancers, especially in colorectal cancer [21]. There were also some reports of mGPS also being prognostic of gastric cancer outcomes $[11,13]$. Interestingly, and in contrast to previous studies, we found that prechemotherapy mGPS was not significantly associated with OS and PFS. Even when categorizing the lower two mGPS scores (o and 1 ) together, survival was not significantly different between either categories $(p=0.100$ and $p=0.134$ for mGPS scores of o or 1 and 2, respectively). A high mGPS score has previously been associated with malnutrition; patients with advanced cancer are often malnourished prior to and during treatment [30]. Tan et al. [31] reported that malnutrition was an independent prognostic factor in patients with cancer, and was also significantly associated with elevated CRP and even high mGPS prior to commencing chemotherapy. The role of malnutrition in patient survival might have outweighed the utility of mGPS as a prognostic factor in our study.

The lack of a correlation between prechemotherapy mGPS and survival in our study is consistent with previously published findings. Dutta et al. [32] suggested that mGPS plus TNM stage could predict prognosis more accurately than mGPS alone in esophageal gastric cancer. Walsh et al. [33] reported that mGPS was not significantly associated with survival in resectable adenocarcinoma of the esophagus or in esophageal-gastric cancer. Similarly, mGPS appears to be of limited use as a prognostic marker in mGC. In our study, the association of survival with postchemotherapy mGPS was different from that of prechemotherapy mGPS in that postchemotherapy mGPS was significantly associated with poor survival $(p<0.001)$. The improved nutritional state of patients after treatment may explain this result [30]. Because the stomach is a key organ in the digestive system, effective chemotherapy might have a positive effect on a patient's nutritional state. As a result, patients receiving effective chemotherapy as well as better nutrition ought to have a low mGPS after chemotherapy, translating into better survival.

Recent studies have clarified the mechanism of suppression of tumor immunity by myeloid-derived suppressor cells (MDSCs) [34]. MDSC levels are higher in cancer patients than in healthy subjects; this is especially true for cancers of the digestive system [35]. MDSCs 
can disrupt immune surveillance against cancer cells and induce cancer progression by immune suppression [34]. Therefore, it could be beneficial to inhibit the factors that induce MDSCs when administering immunotherapy [34]. MDSCs have a positive correlation with absolute neutrophil count and NLR, and a negative correlation with lymphocyte count [35]. In future, it may be prudent to evaluate NLR together with MDSC levels as posttreatment prognosticators; consequently, it will be necessary to identify methods for targeting and inhibiting MDSCs. Meanwhile, we maintain that NLR is useful for measuring treatment response and identifying patients who may be eligible for novel therapies.

In conclusion, prechemotherapy and postchemotherapy NLR values were strongly associated with survival outcomes in mGC; moreover, NLR change patterns were correlated with survival and chemotherapy response. Poor outcomes were observed concomitant with NLRs shifting toward higher values. Therefore, prechemotherapy NLR may be utilized as a prognostic factor in mGC, and evaluating NLR after chemotherapy can assist in predicting chemotherapeutic responses and prognoses in mGC. Conversely, mGPS was not significantly associated with survival; therefore, prechemotherapy mGPS may be of limited use for predicting prognosis in patients with mGC.

\section{KEY MESSAGE}

1. The neutrophil-to-lymphocyte ratio (NLR) as measured both prechemotherapy and postchemotherapy was strongly associated with survival of metastatic gastric cancer (mGC) patients.

2. Change patterns of NLR before and after chemotherapy were correlated with survival and response to chemotherapy in patients with mGC.

3. The prechemotherapy modified Glasgow prognostic score was not significantly associated with survival of patients with mGC.

\section{Conflict of interest}

No potential conflict of interest relevant to this article was reported.

\section{REFERENCES}

1. Torre LA, Bray F, Siegel RL, Ferlay J, Lortet-Tieulent J, Jemal A. Global cancer statistics, 2012. CA Cancer J Clin 2015;65:87-108.

2. Digklia A, Wagner AD. Advanced gastric cancer: current treatment landscape and future perspectives. World J Gastroenterol 2016;22:2403-2414.

3. Maconi G, Manes G, Porro GB. Role of symptoms in diagnosis and outcome of gastric cancer. World J Gastroenterol 2008;14:1149-1155.

4. Yang D, Hendifar A, Lenz C, et al. Survival of metastatic gastric cancer: significance of age, sex and race/ethnicity. J Gastrointest Oncol 2011;2:77-84.

5. Bang YJ, Van Cutsem E, Feyereislova A, et al. Trastuzum$\mathrm{ab}$ in combination with chemotherapy versus chemotherapy alone for treatment of HER2-positive advanced gastric or gastro-oesophageal junction cancer (ToGA): a phase 3, open-label, randomized controlled trial. Lancet 2010;376:687-697.

6. Wagner AD, Grothe W, Haerting J, Kleber G, Grothey A, Fleig WE. Chemotherapy in advanced gastric cancer: a systematic review and meta-analysis based on aggregate data. J Clin Oncol 2006;24:2903-2909.

7. Koizumi W, Narahara H, Hara T, et al. S-1 plus cisplatin versus $\mathrm{S}-1$ alone for first-line treatment of advanced gastric cancer (SPIRITS trial): a phase III trial. Lancet Oncol 2008;9:215-221.

8. Yamada Y, Higuchi K, Nishikawa K, et al. Phase III study comparing oxaliplatin plus S-1 with cisplatin plus S-1 in chemotherapy-naïve patients with advanced gastric cancer. Ann Oncol 2015;26:141-148.

9. Al-Batran SE, Hartmann JT, Probst S, et al. Phase III trial in metastatic gastroesophageal adenocarcinoma with fluorouracil, leucovorin plus either oxaliplatin or cisplatin: a study of the Arbeitsgemeinschaft Internistische Onkologie. J Clin Oncol 2008;26:1435-1442.

10. Mohri Y, Tanaka K, Toiyama Y, et al. Impact of preoperative neutrophil to lymphocyte ratio and postoperative infectious complications on survival after curative gastrectomy for gastric cancer: a single institutional cohort study. Medicine (Baltimore) 2016;95:e3125.

11. Li QQ, Lu ZH, Yang L, et al. Neutrophil count and the inflammation-based Glasgow prognostic score predict survival in patients with advanced gastric cancer receiving first-line chemotherapy. Asian Pac J Cancer Prev 
2014;15:945-950.

12. Cho IR, Park JC, Park CH, et al. Pre-treatment neutrophil to lymphocyte ratio as a prognostic marker to predict chemotherapeutic response and survival outcomes in metastatic advanced gastric cancer. Gastric Cancer 2014;17:703-710.

13. Jiang $X$, Hiki N, Nunobe $S$, et al. Prognostic importance of the inflammation-based Glasgow prognostic score in patients with gastric cancer. Br J Cancer 2012;107:275-279.

14. Hsieh MC, Wang SH, Chuah SK, Lin YH, Lan J, Rau KM. A prognostic model using inflammation- and nutrition-based scores in patients with metastatic gastric adenocarcinoma treated with chemotherapy. Medicine (Baltimore) 2016;95:e3504.

15. Hsu JT, Liao CK, Le PH, et al. Prognostic value of the preoperative neutrophil to lymphocyte ratio in resectable gastric cancer. Medicine (Baltimore) 2015;94:e1589.

16. Yu L, Lv CY, Yuan AH, Chen W, Wu AW. Significance of the preoperative neutrophil-to-lymphocyte ratio in the prognosis of patients with gastric cancer. World J Gastroenterol 2015;21:6280-6286.

17. Lee SJ, Lee HR, Lee TW, et al. Usefulness of neutrophil to lymphocyte ratio in patients with chronic obstructive pulmonary disease: a prospective observational study. Korean J Intern Med 2016;31:891-898.

18. Grivennikov SI, Greten FR, Karin M. Immunity, inflammation, and cancer. Cell 2010;140:883-899.

19. Coussens LM, Werb Z. Inflammation and cancer. Nature 2002;420:860-867.

20. Balkwill F, Mantovani A. Inflammation and cancer: back to Virchow? Lancet 2001;357:539-545.

21. Kishiki T, Masaki T, Matsuoka H, et al. Modified Glasgow prognostic score in patients with incurable stage IV colorectal cancer. Am J Surg 2013;206:234-240.

22. Schwartz LH, Bogaerts J, Ford R, et al. Evaluation of lymph nodes with RECIST 1.1. Eur J Cancer 2009;45:261267.

23. Hanahan D, Weinberg RA. Hallmarks of cancer: the next generation. Cell 2011;144:646-674.

24. Fang H, Declerck YA. Targeting the tumor microenvironment: from understanding pathways to effective clinical trials. Cancer Res 2013;73:4965-4977.

25. Dumitru CA, Lang S, Brandau S. Modulation of neutrophil granulocytes in the tumor microenvironment: mechanisms and consequences for tumor progression. Semin Cancer Biol 2013;23:141-148.

26. Smyth MJ, Dunn GP, Schreiber RD. Cancer immunosurveillance and immunoediting: the roles of immunity in suppressing tumor development and shaping tumor immunogenicity. Adv Immunol 2006;90:1-50.

27. Im SA, Lee KE, Nam E, et al. The prognostic significance of the overexpression of HER-2/neu in Korean gastric carcinomas and the in vitro effects of anti-HER-2/neu antibody on cell growth in the gastric carcinoma cell lines. Cancer Res Treat 2003;35:109-116.

28. Song Y, Huang J, Wang JW. Relationship between HER2/ neu gene amplification and protein expression and prognosis in patients with advanced gastric carcinoma. Chin J Cancer 2010;29:76-81.

29. Forrest LM, McMillan DC, McArdle CS, Angerson WJ, Dunlop DJ. Evaluation of cumulative prognostic scores based on the systemic inflammatory response in patients with inoperable non-small-cell lung cancer. $\mathrm{Br} \mathrm{J}$ Cancer 2003;89:1028-1030.

30. Choi WJ, Kim J. Nutritional care of gastric cancer patients with clinical outcomes and complications: a review. Clin Nutr Res 2016;5:65-78.

31. Tan CS, Read JA, Phan VH, Beale PJ, Peat JK, Clarke SJ. The relationship between nutritional status, inflammatory markers and survival in patients with advanced cancer: a prospective cohort study. Support Care Cancer 2015;23:385-391.

32. Dutta S, Al-Mrabt NM, Fullarton GM, Horgan PG, McMillan DC. A comparison of POSSUM and GPS models in the prediction of post-operative outcome in patients undergoing oesophago-gastric cancer resection. Ann Surg Oncol 2011;18:2808-2817.

33. Walsh SM, Casey S, Kennedy R, Ravi N, Reynolds JV. Does the modified Glasgow prognostic score (mGPS) have a prognostic role in esophageal cancer? J Surg Oncol 2016;113:732-737.

34. Ostrand-Rosenberg S, Sinha P. Myeloid-derived suppressor cells: linking inflammation and cancer. J Immunol 2009;182:4499-4506.

35. Ohki S, Shibata M, Gonda K, et al. Circulating myeloid-derived suppressor cells are increased and correlate to immune suppression, inflammation and hypoproteinemia in patients with cancer. Oncol Rep 2012;28:453-458. 
KJIM'

Supplementary Table 1. Univariate and multivariate analyses for prechemotherapy PFS

\begin{tabular}{|c|c|c|c|c|c|c|}
\hline \multirow{2}{*}{ Variable } & \multicolumn{3}{|c|}{ Univariate } & \multicolumn{3}{|c|}{ Multivariate } \\
\hline & $\mathrm{HR}$ & $95 \% \mathrm{CI}$ & $p$ value & HR & $95 \% \mathrm{CI}$ & $p$ value \\
\hline \multicolumn{7}{|l|}{ Age, yr } \\
\hline$<65$ & 1.00 & & & & & \\
\hline$\geq 65$ & 1.06 & $0.87-1.28$ & 0.589 & & NS & \\
\hline \multicolumn{7}{|l|}{ Sex } \\
\hline Female & 1.00 & & & & & \\
\hline Male & 1.18 & $0.97-1.43$ & 0.091 & & NS & \\
\hline \multicolumn{7}{|l|}{ Detecting metastasis } \\
\hline Recurrence & 1.00 & & & 1.00 & & \\
\hline Initial diagnosis & 1.49 & $1.21-1.84$ & $<0.001$ & 1.36 & $1.10-1.69$ & 0.005 \\
\hline \multicolumn{7}{|l|}{ Histological type } \\
\hline Differentiated & 1.00 & & & 1.00 & & \\
\hline Undifferentiated & 1.28 & $1.05-1.55$ & 0.017 & 1.33 & $1.09-1.63$ & 0.005 \\
\hline \multicolumn{7}{|c|}{ Peritoneal carcinomatosis } \\
\hline No & 1.00 & & & & & \\
\hline Yes & 1.20 & $1.00-1.45$ & 0.055 & & NS & \\
\hline \multicolumn{7}{|l|}{ No. of metastatic organs } \\
\hline 1 & 1.00 & & & 1.00 & & \\
\hline$\geq 2$ & 1.58 & $1.30-1.92$ & $<0.001$ & 1.48 & $1.21-1.81$ & $<0.001$ \\
\hline \multicolumn{7}{|l|}{ First line chemotherapy } \\
\hline Cisplatin based & 1.00 & & & & & \\
\hline Non-cisplatin based & 1.02 & $0.81-1.29$ & 0.850 & & NS & \\
\hline \multicolumn{7}{|l|}{ Prechemotherapy NLR } \\
\hline$<3.0$ & 1.00 & & & 1.00 & & \\
\hline$\geq 3.0$ & 1.37 & $1.13-1.66$ & 0.002 & 1.42 & $1.17-1.73$ & $<0.001$ \\
\hline \multicolumn{7}{|c|}{ Prechemotherapy mGPS } \\
\hline Low & 1.00 & & & & & \\
\hline High & 1.21 & $0.94-1.56$ & 0.131 & & NS & \\
\hline
\end{tabular}

PFS, progression-free survival; HR, hazard ratio; CI, confidence interval; NS, not significant; NLR, neutrophil-to-lymphocyte ratio; mGPS, modified Glascow prognostic score. 
Nam YJ, et al. Inflammatory markers in gastric cancer

Supplementary Table 2. Univariate analysis for OS and PFS by the Kaplan-Meier method

\begin{tabular}{|c|c|c|c|c|c|c|}
\hline Variable & $\mathrm{OS}$, mon, median $\pm \mathrm{SD}$ & $95 \%$ CI, mon & $p$ value & PFS, mon, median \pm SD & $95 \%$ CI, mon & $p$ value \\
\hline Prechemotherapy NLR & & & 0.002 & & & 0.002 \\
\hline$\leq 3.0$ & $14.90 \pm 0.94$ & $13.06-16.75$ & & $7 \cdot 50 \pm 0.37$ & $6.78-8.23$ & \\
\hline$>3.0$ & $11.47 \pm 0.68$ & $10.14-12.79$ & & $5.07 \pm 0.43$ & $4.22-5.91$ & \\
\hline Prechemotherapy mGPS & & & 0.139 & & & 0.129 \\
\hline Low $^{\mathrm{a}}$ & $13.63 \pm 0.66$ & $12.34-14.92$ & & $7.13 \pm 0.36$ & $6.43-7.84$ & \\
\hline High $^{\mathrm{b}}$ & $11.20 \pm 1.13$ & $8.99-13.41$ & & $5.07 \pm 0.67$ & $3.76-6.37$ & \\
\hline
\end{tabular}

OS, overall survival; PFS, progression-free survival; SD, standard deviation; CI, confidence interval; NLR, neutrophil-to-lymphocyte ratio; mGPS, modified Glasgow prognostic score.

${ }^{a}$ Low in mGPS are o or 1 scores.

${ }^{\mathrm{b}}$ High in mGPS is 2 score. 
Supplementary Table 3. Univariate analysis for OS and PFS by the Kaplan-Meier method

\begin{tabular}{|c|c|c|c|c|c|c|}
\hline Variable & $\begin{array}{c}\text { OS, mon, } \\
\text { median } \pm \mathrm{SD}\end{array}$ & $95 \%$ CI, mon & $p$ value & $\begin{array}{c}\text { PFS, mon, } \\
\text { median } \pm \text { SD }\end{array}$ & $95 \%$ CI, mon & $p$ value \\
\hline Postchemotherapy NLR & & & $<0.001$ & & & $<0.001$ \\
\hline$<3.00$ & $14.90 \pm 0.78$ & $13 \cdot 37-16.44$ & & $7.53 \pm 0.29$ & $6.97-8.10$ & \\
\hline$\geq 3.00$ & $8.73 \pm 0.44$ & $7.88-9.59$ & & $3.33 \pm 0.16$ & $3.02-3.65$ & \\
\hline Postchemotherapy mGPS & & & $<0.001$ & & & $<0.001$ \\
\hline Low $^{a}$ & $14.33 \pm 0.62$ & $13.12-15.55$ & & $7.33 \pm 0.30$ & $6.75-7.92$ & \\
\hline High $^{b}$ & $8.23 \pm 0.66$ & $6.93-9.53$ & & $3.17 \pm 0.30$ & $2.57-3.76$ & \\
\hline
\end{tabular}

OS, overall survival; PFS, progression-free survival; SD, standard deviation; CI, confidence interval; NLR, neutrophil-to-lymphocyte ratio; mGPS, modified Glasgow prognostic score.

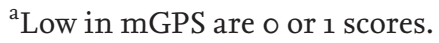

${ }^{\mathrm{b}}$ High in mGPS is 2 score. 
Nam YJ, et al. Inflammatory markers in gastric cancer

Supplementary Table 4. Univariate analysis for OS and PFS according to the change patterns of inflammatory markers

\begin{tabular}{|c|c|c|c|c|c|c|}
\hline \multirow{2}{*}{ Variable } & \multicolumn{3}{|c|}{ OS } & \multicolumn{3}{|c|}{ PFS } \\
\hline & $\mathrm{HR}$ & $95 \% \mathrm{CI}$ & $p$ value & $\mathrm{HR}$ & $95 \% \mathrm{CI}$ & $p$ value \\
\hline NLR change pattern & & & $<0.001$ & & & $<0.001$ \\
\hline Low to low & 1.00 & & & 1.00 & & \\
\hline High to low & 1.26 & $1.00-1.57$ & 0.046 & 1.27 & $1.02-1.60$ & 0.035 \\
\hline Low to high & 1.79 & $1.35^{-2.38}$ & $<0.001$ & 2.14 & $1.61-2.85$ & $<0.001$ \\
\hline High to high & 3.03 & $2.19-4.21$ & $<0.001$ & 3.87 & $2.77-5.40$ & $<0.001$ \\
\hline mGPS change pattern & & & $<0.001$ & & & $<0.001$ \\
\hline Low to low & 1.00 & & & 1.00 & & \\
\hline High to low & 1.05 & $0.79-1.39$ & 0.731 & 1.04 & $0.78-1.38$ & 0.792 \\
\hline Low to high & 1.46 & $1.10-1.96$ & 0.010 & 1.61 & $1.20-2.15$ & 0.001 \\
\hline High to high & 4.14 & $2.52-6.80$ & $<0.001$ & 6.94 & $4.18-11.52$ & $<0.001$ \\
\hline
\end{tabular}

OS, overall survival; PFS, progression-free survival; HR, hazard ratio; CI, confidence interval; NLR, neutrophil-to-lymphocyte ratio; mGPS, modified Glasgow prognostic score. 
Supplementary Table 5. Univariate analysis for OS and PFS in HER2-negative and -positive by the Kaplan-Meier method

\begin{tabular}{|c|c|c|c|c|c|c|}
\hline Variable & $\begin{array}{c}\text { OS, mon, } \\
\text { median } \pm \mathrm{SD}\end{array}$ & $95 \%$ CI, mon & $p$ value & $\begin{array}{c}\text { PFS, mon, } \\
\text { median } \pm \mathrm{SD}\end{array}$ & $95 \%$ CI, mon & $p$ value \\
\hline \multicolumn{7}{|l|}{ HER2-negative $^{a}$} \\
\hline Prechemotherapy NLR & & & 0.002 & & & 0.006 \\
\hline$\leq 3.0$ & $12.13 \pm 0.88$ & $10.40-13.87$ & & $6.53 \pm 0.70$ & $5.16-7.91$ & \\
\hline$>3.0$ & $9.30 \pm 0.78$ & $7 \cdot 77-10.83$ & & $4.43 \pm 0.91$ & $2.66-6.20$ & \\
\hline \multicolumn{7}{|l|}{ HER2-positive ${ }^{b}$} \\
\hline Prechemotherapy NLR & & & 0.105 & & & 0.450 \\
\hline$\leq 3.0$ & $15.73 \pm 1.65$ & $12.51-18.96$ & & $8.73 \pm 1.11$ & $6.55^{-10.91}$ & \\
\hline$>3.0$ & $13.53 \pm 3.56$ & $6.55^{-20.52}$ & & $6.20 \pm 3.02$ & $0.29-12.11$ & \\
\hline
\end{tabular}

OS, overall survival; PFS, progression-free survival; HER, human epidermal growth factor receptor; SD, standard deviation; CI, confidence interval; NLR, neutrophil-to-lymphocyte ratio.

${ }^{a}$ There were 161 HER2-negative patients. Among them 109 patients were NLR $\leq 3.0$ (102 patients died) and 52 patients were $>3.0$ (48 patients died).

${ }^{\mathrm{b}}$ There were 32 HER2-positive patients. Among them 19 patients were NLR $\leq 3.0$ (18 patients died) and 13 patients were NLR > 3.0 (11 patients died). 


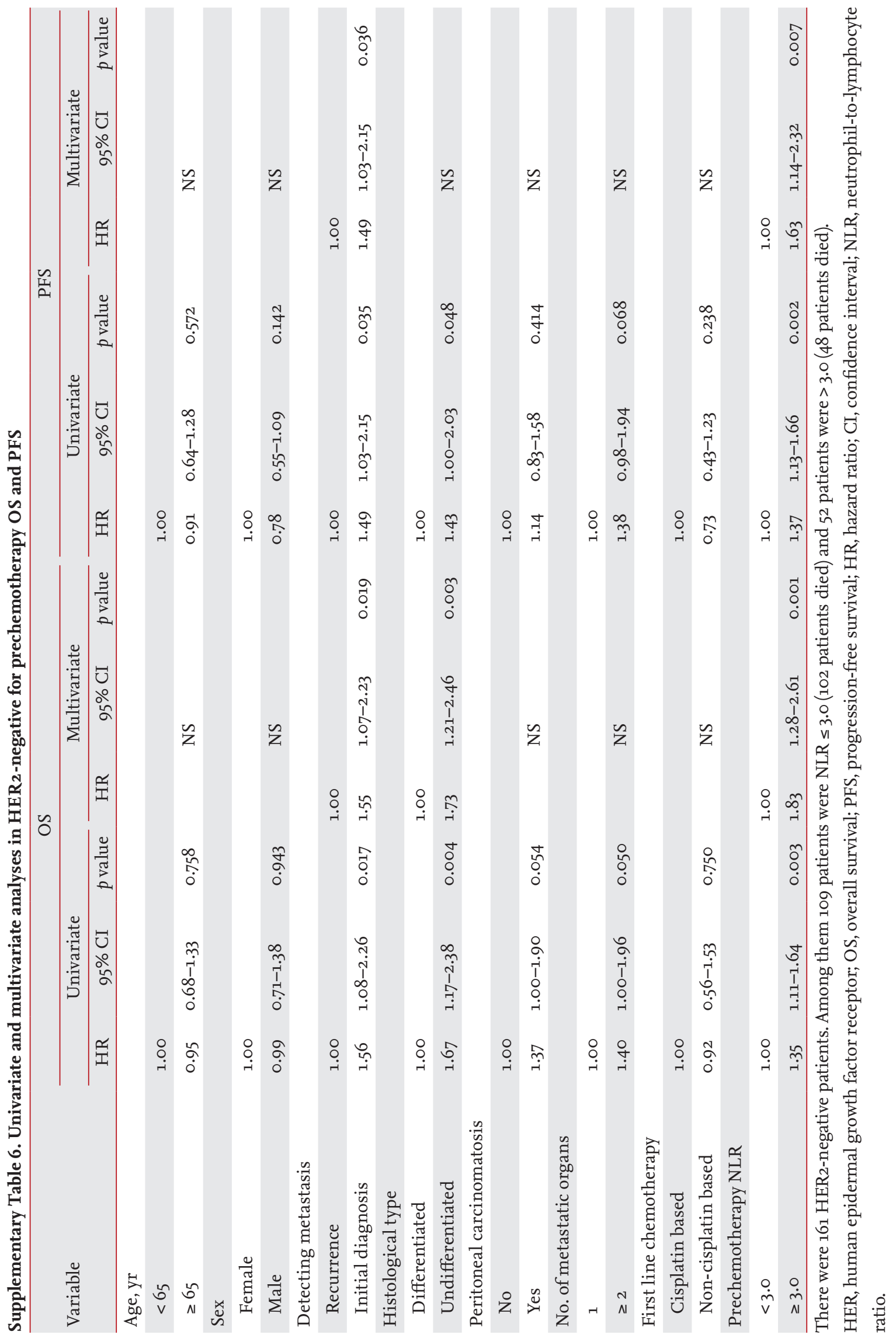



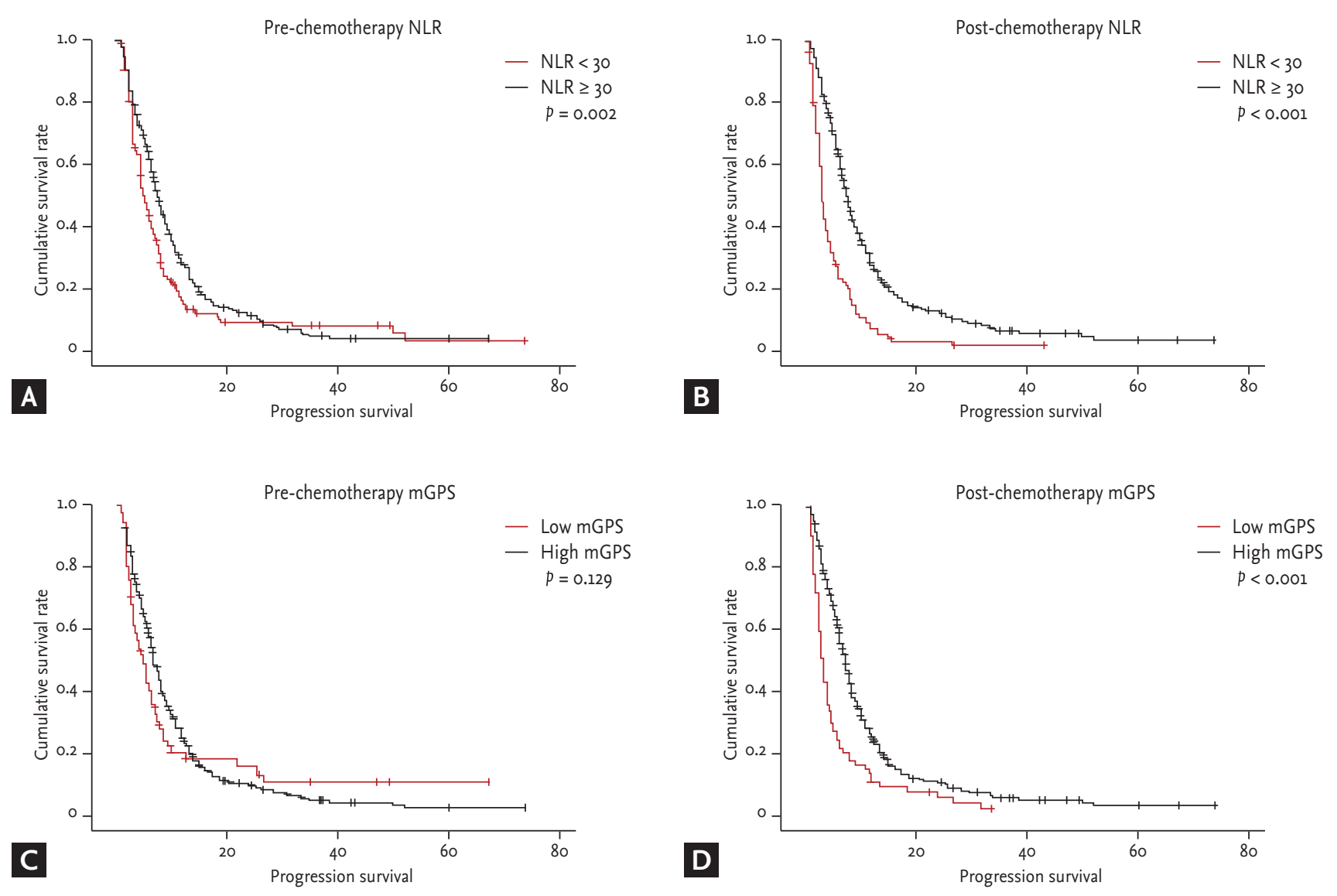

Supplementary Figure 1. Survival curves depending on the inflammatory markers, obtained by using the Kaplan-Meier method. (A) Prechemotherapy neutrophil-to-lymphocyte ratio (NLR). (B) Postchemotherapy NLR. (C) Prechemotherapy modified Glasgow prognostic score (mGPS). (D) Postchemotherapy mGPS. 